Cinémas

Revue d'études cinématographiques

Journal of Film Studies

\title{
Poésie et réalité, le sens caché de L'Oumigmag de Pierre Perrault
}

\section{Claude Albert}

Volume 5, numéro 3, printemps 1995

Cinélekta 1

URI : https://id.erudit.org/iderudit/1001149ar

DOI : https://doi.org/10.7202/1001149ar

Aller au sommaire du numéro

Éditeur(s)

Cinémas

ISSN

1181-6945 (imprimé)

1705-6500 (numérique)

Découvrir la revue

Citer cet article

Albert, C. (1995). Poésie et réalité, le sens caché de L'Oumigmag de Pierre Perrault. Cinémas, 5(3), 101-114. https://doi.org/10.7202/1001149ar
Résumé de l'article

La recherche d'une information connue a priori nous empêche de regarder la réalité telle qu'elle est. Dans le cinéma documentaire, cela donne lieu à une stratégie discursive allant à l'encontre de la recherche d'objectivité. Peut-être passerions-nous à côté de l'essentiel en croyant que L'Oumigmag (1993) porte vraiment sur le boeuf musqué : il s'agit, en fait, d'un essai sur le sens d'un animal emblématique, authentique et marginal qui s'inscrit dans la lignée des Alexis Tremblay, Hauris Lalancette et Didier Dufour. Dans le film, l'auteur y soutient la thèse d'une distorsion de la connaissance par la culture, tout en convoitant l'innocence du regard du chasseur primitif. Sous forme de métaphores, l'interprète se voit soumettre une série d'hypothèses destinées à percer l'énigme des méfiances, de la sauvagerie et des cornes. Reste à savoir jusqu'à quel point le procédé atteint son but, tout indiquant que le projet était d'avance voué à l'échec. 


\title{
Poésie et réalité, le sens caché de L'Oumigmag de Pierre Perrault
}

\section{Claude Albert}

\begin{abstract}
RÉSUME
La recherche d'une information connue a priori nous empêche de regarder la réalité telle qu'elle est. Dans le cinéma documentaire, cela donne lieu à une stratégie discursive allant à l'encontre de la recherche d'objectivité. Peut-être passerions-nous à côté de l'essentiel en croyant que L'Oumigmag (1993) porte vraiment sur le bœuf musqué: il s'agit, en fait, d'un essai sur le sens d'un animal emblématique, authentique et marginal qui s'inscrit dans la lignée des Alexis Tremblay, Hauris Lalancette et Didier Dufour. Dans le film, l'auteur y soutient la thèse d'une distorsion de la connaissance par la culture, tout en convoitant l'innocence du regard du chasseur primitif. Sous forme de métaphores, l'interprète se voit soumettre une série d'hypothèses destinées à percer l'énigme des méfiances, de la sauvagerie et des cornes. Reste à savoir jusqu'à quel point le procédé atteint son but, tout indiquant que le projet était d'avance voué à l'échec.
\end{abstract}

\section{ABSTRACT}

The search for a priorly known information prevents us from looking at reality as it stands. In documentary cinema, it gives rise to a discursive strategy going against the search for objectivity. We could fail to recognize the main point of the movie if we believed that L'Oumigmag (1993) is really about the muskox. In fact, it is an essay on the meaning of an emblematical, authentical and marginal animal, which is in keeping with the tradition of the Alexis Tremblay, Hauris Lalancette and Didier Dufour. In the movie, the 
author defends the proposition of a distortion of khowledge through culture, while coveting the innocent look of the primitive hunter. In metaphorical form, a series of theories designed to solve the riddle of suspicioun, savagery and horns is submitted to the interpreter. Up to what point did the process achieve its aim remains to be seen, since everything indicated that the project was already doomed to failure.

L'objectivité implique une certaine humilité, à n'en pas douter. L'Oumigmag (1994) n'est pas un film destiné à un auditoire ordinaire, à cette catégorie de gens qui croit en la valeur des jugements a priori; bien au contraire, il s'agit d'un documentaire pour un public qui reconnaît d'emblée le caractère trompeur des apparences. Et pour commencer, peut-être faut-il admettre que ce court métrage ne porte pas vraiment sur le bœuf musqué : c'est la valeur symbolique de l'animal qui se trouve dans la mire de la caméra, comme point de départ d'une réflexion philosophique qui ne se résume pas seulement à une esthétique du Grand Nord. Au-delà du message littéral se trouve une théorie du "cinéma-vérité", comme il n’y en avait jamais eu jusqu'à ce jour dans toute la filmographie de Pierre Perrault, ce qui confere évidemment à cette réalisation une importance particulière.

Cette œuvre pose cependant sans ambages un certain nombre de conditions, parmi lesquelles l'" innocence " du regard figure en tout premier lieu. Le spectateur ne peut pas visionner L'Oumigmag sans laisser de côté ses préjugés, ses conditionnements culturels et ses habitudes d'interprétation au risque de se méprendre sur le véritable objet porté à l'écran. Mais n'est-ce pas là une exigence démesurée? Peut-être, à en croire le ton du commentaire, qui accepte d'avance l'échec de l'aventure documentaire. Y aurat-il un public pour ce genre de défi ? L'auteur se prend à en douter, étant donné la force des attentes et la difficulté de voir le monde en face, en tirant le voile du désir et de l'intérêt:

Est-ce que je m'illusionne à attendre un lecteur d'images ayant parcouru d'autres lieux que la fable? Est-ce trop espérer? J'ai beaucoup investi dans un cer- 


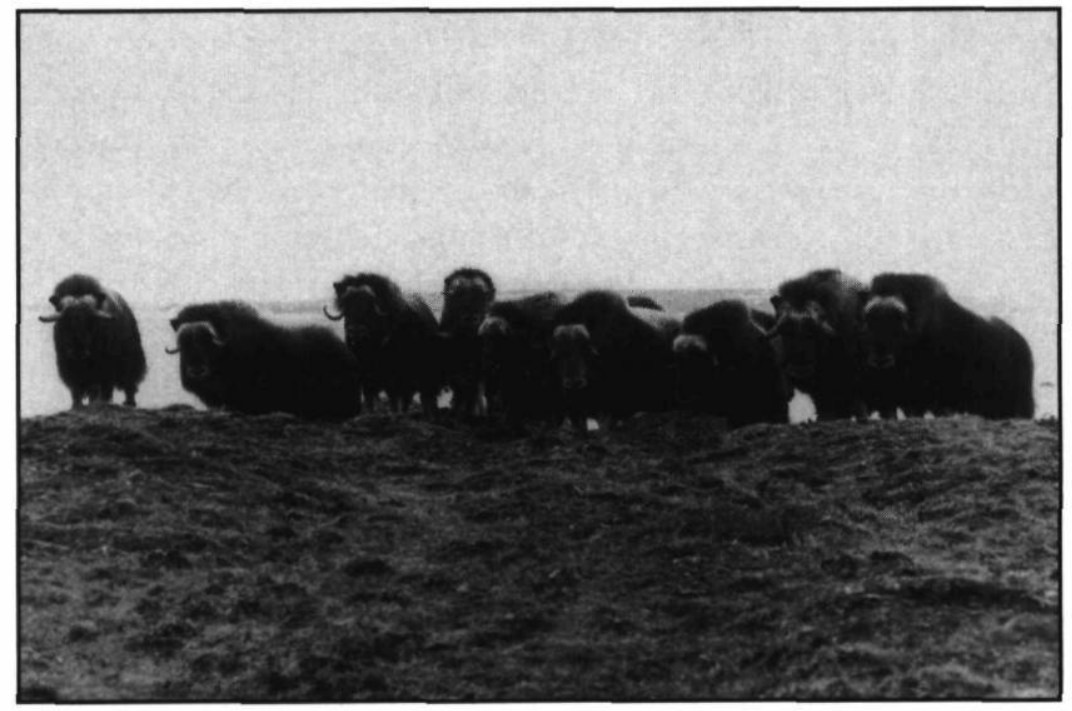

\section{L'Oumigmag de Pierre Perrault (1993)}

\section{Collection ONF}

tain regard. Celui qui refuse de falsifier. Celui qui me sert à lire Cartier. $\AA$ lire le fleuve à mon tour avec les yeux de Cartier. A lire le bœuf musqué, ce soir qui ne tombe pas sur la nuit. Existe-t-il, ce lecteur d'image qui n'attend pas la fable ${ }^{1}$ ?

Mais comment faire abstraction de ce type de conditionnement en sachant, par ailleurs, qu'il détermine nos réactions sensorielles et cognitives les plus élémentaires? Prenons, par exemple, une photographie de famille qui représente de nombreuses personnes. Parmi celles qui y figurent, dans un coin ou au milieu, en avant-plan ou en arrière-plan, à gauche ou à droite, de profil ou de face se détache nettement une physionomie, jamais la même dépendant de qui regarde, et qui en dit long sur la constitution de la pensée. Sur qui l'attention de l'observateur se portera-t-elle en premier? Sur les gens qu'il connaît ou fréquente le plus, bien entendu, puisque notre esprit a toujours tendance à rechercher des signes familiers, mais surtout sur luimême, le soi restant toujours un centre d'attraction privilégié.

Cet égocentrisme est-il une réaction universelle? Ne pas l'admettre, par principe ou pour toute autre raison, contrevient 
aux lois de la psychologie et se présente peut-être comme l'attitude la plus contraire à celle de Pierre Perrault. Il apparaît difficile, en effet, de dénier que des influences inconscientes dirigent notre regard et que cette propension à rechercher les choses et les êtres proches conditionne l'ensemble de nos perceptions. La sensation de "connaissance", qui amenuise la crainte, le risque et le doute, provient de l'observation d'une contingence sélectionnée à dessein et s'accroît dans la mesure où une infinité de détails, pour ne pas dire l'individualité des "phénomènes" au sens philosophique du terme, demeure gravée dans la mémoire. Ce savoir détermine à son tour des habitudes d'interprétation qui nous dissuadent de sortir des sentiers battus: l'inexplicable et l'inconnu génèrent un inconfort qui n'a rien d'invitant et dont il vaut mieux se détourner tant que cela demeure possible. Devenant à la longue bien plus un réflexe qu'une pensée délibérée, cette réaction de protection, dans certaines circonstances et sous la poussée de l'évidence, peut même nous inciter à déformer un réel inconvenant, ce qui démontre que l'acuité du regard se place alors sous la tutelle de la subjectivité. En fait, disposons-nous réellement d'une faculté de percevoir directement ce qui se passe autour de nous, voire d'un pouvoir d'introspection objectif qui s'applique à notre propre personne? Rien de moins sûr, quand on connaît l'extrême pouvoir de mystification de la pensée qui, ne l'oublions pas, forme un relais obligé entre l'information recueillie par les sens et la conscience de chaque individu.

En d'autres mots, c'est un peu comme si la singularité du déjà-connu, qu'il prenne l'allure de l'expérience préalable de l'interprète ou du milieu culturel dans lequel il évolue, retenait le regard de s'ouvrir à ce qui se passe vraiment autour de lui. L'Oumigmag se propose justement de redécouvrir l'objectivité, de nommer le réel en marge de l'action des a priori, projet qui requiert une infinité de précautions. L'image, tant qu'elle ne devient pas discours, s'avère une preuve indiscutable qu'un événement s'est produit dans un lieu et à un temps déterminés, propriété qui, exploitée correctement, a permis l'avènement du cinéma documentaire. Mais il reste que sous cette forme, son expressivité ne peut dépasser un certain niveau: 
Nous avons rempli nos cales de mémoire pour recommencer le voyage au retour. Pour témoigner. Pour restaurer l'instant. Pour revivre l'image comme une mémoire et tout ce qui se passait au-delà de l'image [...] Car l'image contient beaucoup plus que l'image pour ceux qui ont fait le voyage. Et c'est ce que nous avons voulu partager par le récit. Cet invisible que seule la parole peut traduire.

Conscient de ce phénomène, Pierre Perrault ne se fait pas trop de scrupules à prendre la parole, même si son caractère essentiellement symbolique lui confere une plus grande arbitrarité qu'à l'image ${ }^{2}$ : en plus de narrer les pérégrinations du bœuf musqué, il est en train de préparer un ouvrage qui modifiera encore plus la proportion entre l'image et la parole ${ }^{3}$. Cette extrapolation du film, adoptant la forme d'un discours poétique, vise à affiner la sensibilité, à dénoncer l'impact de la fable des cultures et à magnifier la vérité de l'objet. Mais y réussira-t-il sans se faire prendre à son propre jeu? La subjectivité ne risquet-elle pas de s'infiltrer et de reprendre le dessus?

D'en dire et d'en écrire aussi long sur un court métrage de vingt-huit minutes a certainement chambardé notre compréhension de l'Oumigmag. Dans la bonne ou la mauvaise direction, cela dépend de l'importance accordée à l'objectif documentaire. Les prédicats se pressent pour rendre compte de l'image, pour appuyer la succession de l'image, qui constitue déjà une première forme de manipulation. Ce faisant, ils ne se mettent pas forcément au service de la subjectivité, comme le recours systématique à la métaphore pourrait le laisser croire. Dans la mesure où le montage, le bruitage, la musique et la narration concourent à valoriser la relation de fait entre la lumière, l'objet et la pellicule, tout indique que rien de tel ne se produit. Mais aussitôt que l'auteur s'autorise à créer d'autres rapports entre le bœuf musqué et la personne qui le regarde, il en va tout autrement. La difficulté consiste à démêler les passages où il vise à faire apparaître des aspects de l'événement imperceptibles au premier chef de ceux où il introduit des éléments étrangers à l'observation.

Le film de Perrault se déploie d'une manière qui reflète fidèlement ses inquiétudes: d'une part, il expose ses vues sur le signe 
du réel figuré par l'animal, sous forme de questions, d'hypothèses et de spéculations; d'autre part, sur un ton plus affirmatif, il essaie de justifier l'objectivité de sa démarche tant sur le plan du "caméramage" que de son exploitation philosophique. C'est un peu comme s'il sentait le besoin de mettre en évidence le caractère énigmatique de l'Oumigmag, mais en même temps la détermination et la confiance de percer son secret en adoptant une méthode rigoureuse.

D'emblée, nous assistons à un aveu d'ignorance quant à la nature de la sauvagerie qui se profile au-devant des lentilles et qui semble porteuse d'un savoir absolument réfractaire à l'ascendant des cultures. Qu'est-ce que le ruminant authentique quand il ignore la présence de l'homme? Difficile à dire :

\begin{abstract}
Aujourd'hui, une petite harde nous permet la proximité. Que savent-ils désormais de nous qui changera leur attitude? En filmant une fleur on peut supposer l'objectivité, présumer de notre absence. Devant les bêtes, on est forcé d'admettre notre existence. Faut-il établir une relation qui risque de se détériorer? Ou se dissimuler ? Faut-il admettre la pénible démarche, l'ignorance, l'impossibilité d'échanger des ambassadeurs, l'absence de connivence?... et se contenter de la tête sculptée de Laugerie, qui ne sera jamais égalée!
\end{abstract}

Tout au plus, en manière de fausse piste, apprenons-nous que la bête est une image de l'" homme approximatif", mais aussi une figure du " lichen tenace en butte à la mort de froid qui voisine le nord du monde". Pourquoi l'avoir choisi comme sujet d'un film documentaire? Parce que le bœuf musqué jouit en toute liberté d'une intelligence de survie absolument extraordinaire, d'une logique de transhumance, de protection, de nutrition et de reproduction instinctive qui témoigne d'une connaissance intime et presque parfaite de son milieu environnant. Il s'agit d'un cas particulièrement flagrant d'ouverture à l'individualité des choses où la précarité de la végétation, la rigueur du climat et la voracité de l'homme n'empêchent aucunement la vie de suivre son cours.

Cette communion avec le réel semble d'ailleurs tellement complète que le temps et l'espace n'exercent apparemment 
aucune emprise sur sa destinée. Depuis environ 12000 ans, le regard et la prudence du bœuf musqué ne se sont pas altérés et recèlent un enseignement qui, au grand dam de l'équipe de tournage, refuse de se donner dans l'immédiat:

Nous sommes, à bien y penser, plus loin dans le temps que dans l'espace. Dans un ailleurs bien sûr qu'on peut situer en longitude et en latitude sur une carte, mais surtout dans le temps, dans un temps sans histoire et qui se mesure en millénaires. Dans la grande écriture des géologies! Parmi les poèmes épiques des glaciations impitoyables! Dans les secrets bien gardés des pollens accumulés! Au beau milieu de l'abondance herbivore!

La continuité de la réflexion de Pierre Perrault est ici remarquable. Dans Les Voiles bas et en travers et La Grande Allure, ses deux derniers films, produits respectivement en 1983 et, en 1985, il avait tenté de refaire le parcours de la découverte de l'Amérique pour réaliser que le pays tel que perçu spontanément par les grands explorateurs n'était en vérité qu'une projection de leurs intentions. Il recommence maintenant en essayant de remonter au-delà des dogmes de la connaissance, où s'éliminent les forces obscures qui conditionnent le regard:

\begin{abstract}
Nous assumons l'ignorance comme condition préalable. Nous avançons dans l'inconnu du silence. À part un bruit d'herbe inaccessible qui rumine. Nulle part ni temple ni divinité. Nulle consultation d'aucune croyance. Nous sommes en toute étrangeté. Sur une terre profane. Bien plus que païenne. Pour ainsi dire, antérieure à la chasse. D'avant les parois. Les cryptes.
\end{abstract}

Les envoyés du roi de France, après avoir refusé d'admettre l'insularité de Cuba et l'humanité des peuplades indigènes, se croyaient vraiment arrivés en Inde parce que le "prince" voulait tant découvrir une nouvelle issue. Pendant ce temps, les troupeaux agglomérés sur les collines à fleur de glacier anticipaient la venue de l'homme blanc dans une attitude bien plus réaliste, celle d'un "racisme d'instinct", dont l'efficacité ne s'est guère démentie. Comment le mettre en joue, ce leste et bel animal qui se fond si bien avec les laineuses altérités du pays? "On dirait 
une parenté entre la bête couverte de son poil et la pierre rongée par le lichen. Une continuité." Pourquoi combattre ce front massif qui vous défie depuis la nuit des temps? "Obstiné, il recommence la tournure, il s'installe dans l'immuable. Il reproduit la lenteur. Il est déjà responsable de tout l'avenir. Il est déjà la conséquence d'un immense passé."

Le chasseur d'images, confondu avec l'ennemi prédateur, se prendra même à douter de l'existence de son vis-à-vis. Malgré tous ses efforts, l'individualité de l'Oumigmag se dérobe obstinément à toute tentative d'élucidation :

\begin{abstract}
L'air d'aller partout. Et nulle part. Sans savoir la différence entre ici et ailleurs. Les bêtes savent-elles où elles vont? Le troupeau va quelque part. On dirait que c'est le troupeau qui existe. Les bêtes filent entre les doigts du troupeau.
\end{abstract}

Pour percer le secret de ces bêtes, il ne faudrait surtout pas lésiner sur les moyens: les observer à bout portant, les suivre, les sentir, apprivoiser leur liberté, prendre contact avec elles, tout ce qui rend la chasse si ardue en raison de la rigueur historique des méfiances, tout ce qui se situe au-delà des classifications scientifiques. "Prendre langue" avec l'animal au contraire de Christophe Colomb qui refusait obstinément de dire ou d'entendre le nom des réalités d'outre-mer.

Bien entendu, les questions s'accumulent et se pressent dans la tête de l'interprète du film. Vulgarisateur de l'image, Pierre Perrault nous les repose sans arrêt comme pour indiquer le sens de l'objectivité. Où va l'Oumigmag? Que cherche-t-il ? Qu'estce qui l'entraîne dans un seul et grand mouvement? Y a-t-il " une loi, une intention, un mythe qui [le] dirige? Les hommes poursuivent les mythes, obéissent aux lois et rêvent de liberté. A quoi rêvent les bœufs musqués " ? Pour y répondre, une seule hypothèse, constamment reformulée, celle de la présence d'une vérité brute, explicite et définie qu'il s'agit de recomposer en dehors de la fable; puis trois avenues de recherche, à caractère fortement spéculatif, qui convergent vers un même but: expliquer la logique du comportement animal, combler la distance entre le cinéaste et le troupeau, entrer en communion avec 
l'intelligence qui le gouverne. Cela est-il concevable en recourant uniquement à la magie des lentilles et à la rigueur discursive? Réussira-t-on jamais à combler le vide creusé par des millénaires de suspicion, à mettre à jour les intentions réciproques, à donner la parole au silence obstiné de la corne et du regard invincible?

Sur le plan de la méthode cinématographique, cette investigation requiert des prises de position résolues, des affirmations tranchées et une éthique à la hauteur de l'objet convoité. Comme à l'accoutumé, Pierre Perrault ne se fait pas prier pour s'affirmer, allant même jusqu'à prêter serment :

En vérité, je vous le dis, tout ceci n'est pas une fable et rien de ce que je vous raconte n'est arrivé ailleurs que dans mes yeux arc-boutés aux lentilles trépidantes quand la nuit tardait à venir parce qu'il restait toutes les fleurs à bâtir avant trop tard.

L'objectivité s'impose avec toutes les exigences qu'elle comporte, en commençant par accepter l'inusité, le marginal, l'inattendu de la découverte. Un simple calque de la réalité ne suffit pas à révéler l'essentiel qui se cache en deçà des apparences. La curiosité du chercheur est mise à contribution, lui qui se doit par avance de renoncer à atteindre son but, visant seulement à s'en approcher. Dans cette optique, désavouant ses attentes, il doit se tenir prêt à respecter un certain nombre de consignes qui constituent la partie catégorique de l'exposé.

Lauteur de films documentaires, en premier lieu, doit renoncer à la fiction qui se propose, à défaut d'une preuve empirique satisfaisante, de suppléer à l'inexplicable. Les mythes, c'est bien connu, compensent pour les incapacités de l'homme, d'où la nécessité de s'en écarter pour renouveler la sensibilité du regard. Dans sa verve habituelle, Pierre Perrault soutient que ce qui demeure, le phénomène incident saisi dans le moment fugace de sa réalisation, exerce une fascination extrême, intrinsèque et inépuisable, idée qu'il prétend être le seul réalisateur québécois à défendre. Mais comment démêler le phénomène brut de sa représentation sémiotique, voilà une question délicate à laquelle L'Oumigmag n'a certainement pas la prétention de répondre. 
Seulement y est-il indiqué avec confiance que la vie a une valeur dans sa manifestation immédiate, individuelle et spontanée. L'auteur n'a certes pas la capacité de s'abstraire complètement du mythe et de la fiction, il en a seulement la ferme intention et adopte les moyens que son "instinct" de cinéaste reconnaît les plus à propos. Avec son équipe, conséquemment, sans aucune garantie, il use de l'optique au gré des événements:

Des gestes indispensables: traîner le trépied, le dresser, chercher le niveau, installer la caméra. Sur un terrain sans abri. Plus rien ne nous protège. Les bêtes sont couchées dans la ravine. [...] Lentille. Ouverture. Mise au point. Foyer. Convergences. Navigations dans l'espace. Véritable navigation. Faire le point. Se situer en rapport à la lumière. Apprécier la distance. Regard mécanique. Naviguer dans l'image. Je reconnais d'avance l'insignifiance de l'image. Et l'impertinence de l'objectivité. Mais l'image et l'objectivité sont voyage et navire. Instruments d'une navigation dont on devra écrire la relation.

Dans les circonstances de l'action immédiate, surtout quand se disputent des enjeux importants, la pensée rationnelle, cohérente et vraisemblable n'a pas une grande efficacité. Devant un troupeau en mouvement qui, dès la première journée en Ungava, se trouve à la portée des caméras, la logique n'a pas beaucoup d'emprise et le souci de composer un discours comme il était globalement prévu cède le pas à la pression des faits.

Tout compte fait, cependant, avec le recul que permet la mémoire cinématographique, les choses finissent toujours par s'arranger. Le montage compense pour les égarements du tournage, introduisant subrepticement une intention discursive. Les seuls gros plans apparaissant dans la première partie du film illustrent ce fait de façon convaincante: présentés en surimpression, au ralenti, ils visent à faire anticiper la clarté des détails que permettront l'approche, fil conducteur et objectif du film. Aussi pouvons-nous légitimement nous interroger sur le "reportage" des faits, qui ne retient peut-être que les séquences pertinentes à la démonstration d'un message alors que le message devrait plutôt se trouver au service de l'image. Mais est-ce bien là ce qui se produit dans L'Oumigmag? 
Cette marginalité obsédante, dont le sens a résisté à quatre mois d'observation, pouvait-elle seulement se livrer par le truchement de la lumière? Pour que s'accomplisse la relation documentaire, comment ne pas y croire un peu et faire en sorte que les procédés du discours accentuent cet effet? Mais que faire quand les circonstances ne suffisent pas à raconter une histoire?

Nous sommes immobiles autour du trépied et de nos manigances comme si l'inaccessible nous affrontait dans toute son ampleur. La réalité qui tant nous sollicite nous fera-t-elle faux bond, nous imposant les subterfuges? Jamais je n'ai autant douté de l'objectivité. Jamais je n'ai autant espéré de la réalité. Est-ce un mauvais calcul?

Lorsque la bête disparaît en ne laissant que des empreintes, le doute s'installe et la nécessité de produire un documentaire aussi bon que possible encourage le factice: "Faudra-t-il à tout prix encore une fois inventer une quelconque divinité pour venir à notre rescousse et donner naissance à la bête séculaire dans la caverne de l'âme occupée par ailleurs d'autres instances?" Assurément, le bœuf musqué, que très peu de Québécois connaissent et associent encore moins à la faune de leur pays, présente assez peu d'intérêt s'il n'est pas interprété de quelque manière. Aussi, en plus de sa présence physique et de son action, il a besoin de l'appui du langage. Reconnaissant les limites de l'image et, même, la subjectivité d'un plan-séquence qui couvrirait toute la durée du film, Pierre Perrault n'a pas le choix d'agir : le réel ineffable, enfermé dans la tournure des cornes et l'intériorité du regard, faudra-t-il, pour le rendre visible, réinventer le médium?

Une connaissance parfaite d'un aspect donné de la contingence est un idéal qui ne sera vraisemblablement jamais atteint. Pour tout savoir de l'Oumigmag, il faudrait tirer une conclusion nécessaire à partir de prémisses entièrement déterminées, c'est-àdire individuelles, ce que les sens ne permettent jamais d'accomplir. Seule une communauté de regards œuvrant de façon désintéressée dans un avenir infiniment long pourrait éventuellement y parvenir, mais jamais dans la ponctualité du moment, ce qui laisse toujours incompris un aspect ou l'autre de ce que nous croyons familier. La régularité du monde frappe immédiatement

Poésie et réalité, le sens caché de L'Oumigmag de Pierre Perrault 
notre attention, reléguant en marge ce qui apparaît de façon fortuite et désordonnée. Cette discrimination n'est pourtant pas infaillible: l'histoire de la recherche scientifique démontre qu'en établissant des rapports en apparence fantaisistes entre des phénomènes inexplicables, seule démarche susceptible de générer des connaissances nouvelles, s'imposent parfois des explications inattendues, qui deviennent aussitôt des habitudes d'interprétation. Avant les théories de Galilée, la thèse de la rotondité de la terre faisait figure de métaphore au même titre que la poésie de Pierre Perrault; il n'empêche qu'elle s'appuyait sur des considérations rationnelles, ce que nous ne dénions pas non plus à la poésie de ce dernier. Le trope accomplit ce que la pensée causale, l'autorité du jugement ou les statistiques sont impropres à réaliser: elle ouvre des avenues d'apprentissage jusque-là ignorées. Il n'y a donc pas de contradiction à en faire le procédé par excellence de l'objectif documentaire, en autant que l'observation serve invariablement à valider les hypothèses qu'elle soulève.

De ce fait, l'appellation de cinéma "direct" devient inappropriée. Il s'agit principalement de mettre la spéculation au service de la réalité, dans l'espoir que se développe ultimement une faculté d'aperception directe, ou du moins "objective", du monde qui nous entoure. À cet égard, Pierre Perrault se situe sans contredit dans une classe à part, n'ayant que très peu cédé en trente ans au mythe et à la fiction. Concrètement, cela l'oblige à adopter une éthique qui a peu de chances de le rendre populaire.

En premier lieu, renoncer de plaire à l'institution cinématographique, elle-même sous la dépendance de la rentabilité commerciale et des intérêts culturels dominants; au contraire, il faut destiner le film documentaire à des gens sensibles à l'altérité des choses, ce qui complique singulièrement l'entreprise. En second lieu, le cinéaste doit se sentir toujours prêt à admettre son erreur et à 's'inscrire en faux contre les valeurs admises, quitte pour cela à faire son deuil du lucre et de la vraisemblance; car le réel finira tôt ou tard par ramener l'imaginaire dans le bon chemin, ce dont il vaut mieux s'aviser rapidement pour éviter de plus grandes déceptions. Troisièmement, puisqu'elles agissent généralement de façon insidieuse, il est préférable de se tenir à l'écart des 
idéologies; gare aux aventuriers qui parcourent le Grand Nord à l'affût de l'exploit, leur ambition déformant le regard au point de le rendre méconnaissable:

Encore aujourd'hui, l'audience est séduite par le pôle. Et chacun à sa manière plante son drapeau sur le sommet du monde à la dérive [...] Et les drapeaux désemparés se promènent sur les glaces emparées par les courants. Encore et toujours le mythe inusable: à chiens, en hélicoptère, caméra vidéo à la main pour s'autofilmer, à pied, à skis, en ultra-léger, en solitaire. Étrange solitude entourée de caméras. L'Arctique n'a-t-il rien d'autre à offrir?

Et le réel dans tout cela? N'y a-t-il pas moyen de s'en préoccuper davantage? Le cinéaste n'a pas de pouvoir de divination et se doit nécessairement de partir de ce qu'il connaît le mieux, les apparences, pour se décharger petit à petit des préconceptions de la fable. Ainsi, le réalisateur de L'Oumigmag se retrouve en position de force, son travail consistant désormais à se préoccuper des allures souvent trompeuses de la lumière, à constituer une mémoire représentative de l'événement, à adopter une poésie visuelle et narrative révélant le phénomène. Pour faire apparaître l'invisible, pour désautomatiser le langage et la pensée, pour "prendre langue" avec le bœuf musqué, qu'y a-t-il d'illicite à repenser la signification des choses? Ne devrions-nous pas y voir une nécessité du cinéma-vérité, un impératif de l'objectif documentaire?

Cette hypothèse, renvoyant sans cesse à une marginalité obsédante, à une authenticité cachée derrière l'évidence, nous a fait voir tour à tour les personnages d'Alexis Tremblay, d'Hauris Lalancette, de Didier Dufour, de Stéphane Albert Boulais, de l'Oumigmag et de bien d'autres, sans jamais déboucher sur une réponse définitive. La méthode d'investigation a beau être claire et affirmative, son objet ne s'en montre pas moins fluctuant et inépuisable. Pierre Perrault se croit trop dépourvu de la faim du chasseur au tambour pour percer l'énigme de la survie millénaire, de la prudence des femelles, du signal de l'attroupement, de l'acuité du regard - malgré la magie du rapprochement optique et le privilège d'une proximité physique éphémère. En 
tout et partout, il n'aura réussi qu'à relater "l'histoire d'une chasse ratée, l'histoire de longs pourparlers qui n'abouti[rent] qu'à une trêve à couteaux tirés ". Mais avec quel brio! L'Oumigmag apparaît comme le résultat d'une enquête ambitieuse, exigeante et délibérée sur un peu plus de trois décennies. Il serait dommage de se méprendre sur ses véritables intentions, lui qui se trouve en attente d'un public désireux de le prolonger dans son propre commentaire.

Université Laval

\section{NOTES}

1 Comme toutes les citations figurant dans cet article, celle-ci provient d'un ouvrage en préparation qui s'intitule L'Oumigmatique ou l'objectif documentaire. Dans le chapitre d'un catalogue publié par l'ONF en 1989 dans le cadre du festival Le Documentaire se fête, Pierre Perrault s'explique sur sa compréhension de l'objectivité et la fonction du film documentaire. À l'instar de Françoise Beaulieu ( $c f$. "L'Oumigmag, ce difficile parcours entre le mot et les choses " dans ce même numéro), je m'en inspire pour étudier la relation entre l'œuvre cinématographique et la réalité qu'elle représente.

2 Voir à ce sujet les deux ouvrages suivants: Ferdinand de Saussure, Cours de linguistique générale (Paris: Payot, 1969) et Michel Foucault, Les Mots et les Choses (Paris: Gallimard, 1966).

3 Voir la note 1. 